\section{§7. Study of an Edge Transport Barrier by Langmuir Probes in the Compact Helical System}

Takeuchi, M. (Dep. of Energy Eng. and Sci., Nagoya Univ.), Toi, K., Nagaoka, K., Suzuki, C., Minami, T., Akiyama, T., Yoshimura, Y., Nishimura, S., Isobe, M., Shimizu, A., Takahashi, C., Matsuoka, K., Okamura, S., CHS Group

Measurements of detailed structure of ETB plasmas are crucial for better understanding of the L-H transition and ETB formation. A triple Langmuir Probe (LP) is well suited for this purpose, because it can measure electron temperature $\left(T_{e}\right)$, density $\left(n_{e}\right)$, space potential $\left(V_{*}\right)$ and their fluctuations in the plasma edge having high time ( $1 \mu \mathrm{s})$ and spatial resolutions. Moreover, measurements of the toroidal uniformity of the ETB structure are particularly important for an ETB plasma in three-dimensional configuration such as CHS. We attempted to measure the ETB structures by using two sets of LPs at two toroidal locations of CHS (the "6U port" and the " $3 \mathrm{U}$ port") as shown in Fig.1. The LP installed at each port section was moved vertically shot by shot from the normalized minor radius $(\rho=r /\langle a\rangle) \rho \sim$ 0.93 to $\rho \sim 1.1$ for about 40 ETB shots with high reproducibility.

Figure 2 shows the radial profiles of $T_{e}, n_{e}$, floating potential $\left(V_{f}\right), V_{s}$ and radial electric field $\left(E_{r}\right)$ measured at the $6 \mathrm{U}$ port section (left) and at the $3 \mathrm{U}$ port section (right), for four time slices averaged over a $1 \mathrm{~ms}$ time window, where " 0 ms" stands for the transition defined by the start time of the drop in the $H_{\alpha}$ emission. At the $6 \mathrm{U}$ port, the radial profile of $T_{e}$ has a hollow structure at $0.95<\rho<0.98$ before the transition, and evolves to the profile with a modest gradient inside $\rho \sim 1.0$ after the transition. Just after the transition $(\mathrm{t}=+4 \sim+5 \mathrm{~ms})$, the radial profile of $n_{e}$ has a steep gradient inside $\rho \sim 0.96$, having a hollow structure at $0.95<\rho<0.98$. After that $(\mathrm{t}=+8 \sim+9 \mathrm{~ms})$, the hollow region of $n_{e}$ is filled from the inner side toward $\rho \sim 0.97$, and at $\mathrm{t}=+16 \sim+17 \mathrm{~ms}$ the hollow structure of $n_{e}$ disappears and is filled up to $\rho \sim 1.0$. This peculiar edge structure may be linked to the presence of a magnetic island at the rotational transform $\imath / 2 \pi=1$, of which the position of the rational surface is calculated to be $\rho \sim 0.95-0.96$. On the other hand, at the $3 \mathrm{U}$ port, $n$, has a steep gradient inside $\rho \sim$ 1 just after the transition. The radial profile of $T_{e}$ is almost fixed across the transition. These observations suggest that the ETB will be formed inside $\rho \sim 1$. However, the initial growth of the ETB in $n$, and $T$ profiles at the $6 \mathrm{U}$ port section may be locally blocked by the presence of a non-rotating magnetic island, which is predicted to be in the region of $0.95<\rho<0.98$ from the shape of the $n_{e}$-profile and to be $\sim 6 \mathrm{~mm}$ width. It is seen from time evolutions of $n$ and $T$ profiles that the island seems to shrink in the deep H-phase after $\sim 15 \mathrm{~ms}$ from the transition.

It is generally thought that $E_{r}$ and its shear $\left(E_{r}^{\prime}\right)$ play an important role in the formation of an ETB. The $E_{r}$ was evaluated from the radial derivative of fitted profiles of $V$. In the $6 \mathrm{U}$ port, $E$, behaves peculiarly having a positive hump for about $10 \mathrm{~ms}$ after the transition in the region of $0.96<\rho<0.99$. Before $(\mathrm{t}=-4 \sim-3 \mathrm{~ms})$ and just after $(\mathrm{t}=$ $+4 \sim+5 \mathrm{~ms}$ ) the transition, the $E_{r}$ shear in the region of 0.94 $<\rho<0.96$ changed largely from $\sim-600 \mathrm{kV} / \mathrm{m}^{2}$ to $\sim 2700$ $\mathrm{kV} / \mathrm{m}^{2}$ and that in the region of $0.98<\rho<1.0$ also changed from $\sim 200 \mathrm{kV} / \mathrm{m}^{2}$ to $\sim-1800 \mathrm{kV} / \mathrm{m}^{2}$. On the other hand, at the $3 \mathrm{U}$ port, the $V$ and $E_{r}$ profiles have a rather monotonic shape without any hollowness or hump. And the $E_{r}$ shear changed from $\sim-200 \mathrm{kV} / \mathrm{m}^{2}$ to $\sim 400 \mathrm{kV} / \mathrm{m}^{2}$ in the region of $0.94<\rho<0.96$.

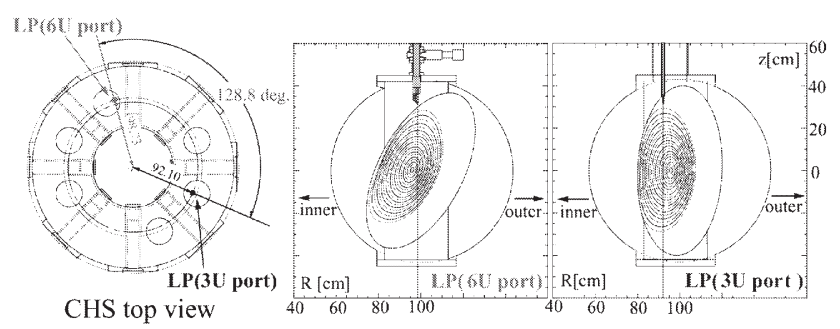

Fig.1 Arrangement of two LPs at two toroidally different sections in CHS (6U and $3 \mathrm{U}$ port sections).
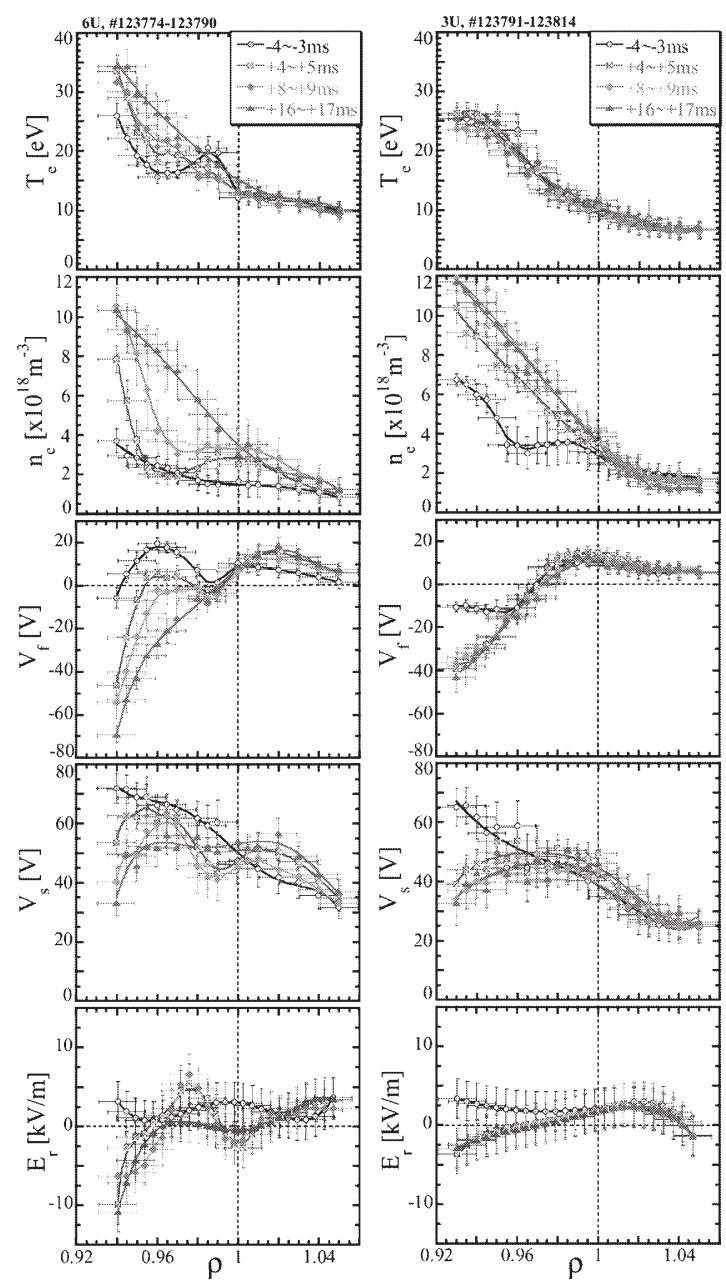

Fig.2 Radial profiles of $T_{e}, n_{e}, V_{f}, V_{s}$ and $E_{r}$ at four time slices averaged for a $1 \mathrm{~ms}$ time window at the $6 \mathrm{U}$ port section (left) and at the $3 \mathrm{U}$ port section 